\title{
日内瓦和平利用原子能会講上提出的 關於中子研究的報告
}

Б. П. 阿傑雅謝維奇

\section{B 雷}

目前只有使用核反应堆才可能獲得用於和本目的的原子能。在核反应堆中淮行着复 雜的物理过程。研究这些过程就所以加深了解与現有反应堆的工作有關的閜題, 以及找 出制成更完善的反应堆的途徑。關於这些过程及有關問題的研究，是会議上提出的報告 的主要部分。

这裏我不想詳尽討論所有提出的關於物理学的研究報告，只想境明一下反映於日内 瓦会議的報告中的在中子物理学領域內的幾个主要研究方向上所獾得的結果。

首先在会議上提出了一系列不同能量的中子和重原子核之問的相互作用的研 究報 告。也提出了大量用各种類型中子譜儀量度不同能量的中子和分致元素核之間相互作用 的截面的報告。中子和原子堆構造材料的原子核之間的相互作用也具有不小的意义. 大 家都知道，在核分裂時放出一定數目的次級中子，十分㫾顯，这个数目对於反应堆中發 生鋋式反应的可能性來說有着基本的意义。很多報告討論这个問題以及与此相联系的另 一个問題, 即每次俘獲一个中子所座生的次級中子數同被俘中子能量之間的關係的問 題。所謂楥發中子对於核反应堆中鐩式反应的動力学起着很大的作用。也有一些關於这

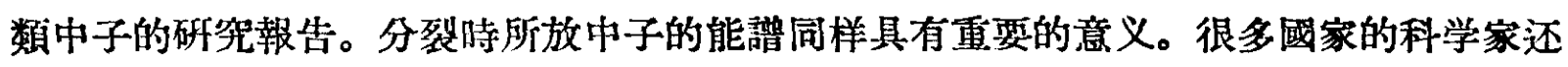
研究了各种材料的減速性質, 在这些研究中既使用了大家早巳知道的研究中子在減速剂 中穞定分㛂的方法, 也使用了脈冲式中子源的新的動力学方法。会議对鈾 238 中子共振 吸收的研究給予了很大的注意。因为利用原子能之所以可能, 主要是由於核的分裂的性

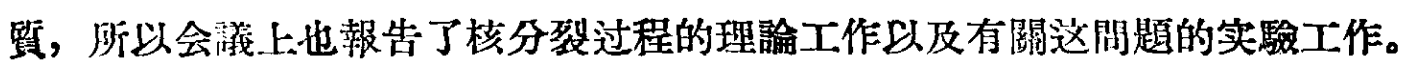

核反应堆屏生了强中子束, 因此就有可能來研究中子的 $\beta$ 蛻变問題, 研究原子核保 獾中子後的 $\gamma$ 射線, 以及一系列有關固体理論的問題。

顯然会議上所討論的問題不僅有实用的性質, 而且有些問題也有独立的学術上的價 值, 它們有助於更深入了解原子核中進行的过程以及研究它們的楎造。

此外在会議上所提出的侼告中还描迅了很多新的已經用來做实驗工作的实驗設备. 同時也閶明了各國有關中子物理学的基本工作方向。

2 月号科学通报 


\section{中于错啭}

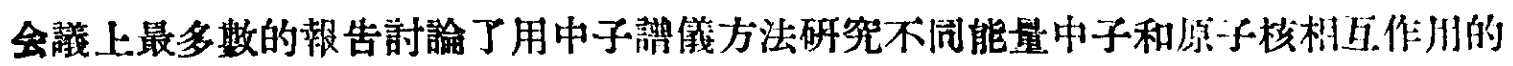
問題。中子譜儀可以用來研究选定的能量範图中的中子同原子核的相互作用。用川子端 儀可以確定相互作用的總有效截面，以及分裂截面、俘獾截面等。此外，中子洋儀批够 用來量度从分裂的核放出來多少次級中子，以及研究中子和固体結晶點泍間的相互作用

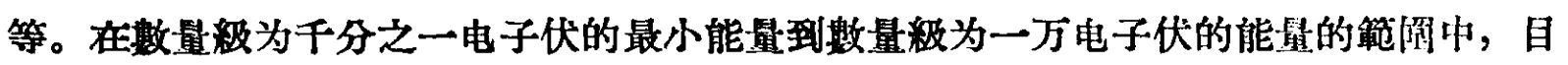
前主要使用二种類型的諳儀。第一种是晶体單色器, 它的原理是用晶体反射作㳉來选擇 一定能量中子。第二种是按照飛越時間來选擇一定能量的中子，会議上所提出的媇少， 主要描迅按照飛越時間选擇原理工作的各种類型的㦈儀。按飛躍時間來洗擇中子的找術 被应用在利用帶电粒子咅生的脈冲中子源上，她被应用在由反应堆發出的脈冲中子束.上。 为了从反应堆走生中子脈冲, 应用了机械截断器。

按速度选擇中子的基本原理与中子脈冲如何道生並没有䦥係。应該注意, 按飛躍時 間选擇中子的方法僅僅分出具有一定速度范圍的中子，而不是得到單一能显的中子。从 另一方面峵，它也有長处，可以同時記錄各种中子能量萣中大量（１00）中子。

在反应堆發出的很强的熱中子束和共振中子束的利用方面, 按飛䠰時間选擇中子的 方法得到了度泛的应用。这个方法的成就是很大的, 關於能量一万电子伏以下的中子的 反应截面的基本知識就是利用这个方法得到的。中子能䜊方法相当久以前就知道了。这 种譜儀有成效地被用來測量熱中子同原子核的相互作用的截面。另一方面利用像静电加 速器一類的加速器也成功地得到了能量約一万到两万电子伏的中子。但是这个方法的分 辨率在低能筑圈內很快地降低, 各个國家的在这方面工作的学者都在努力改進諨儀的分

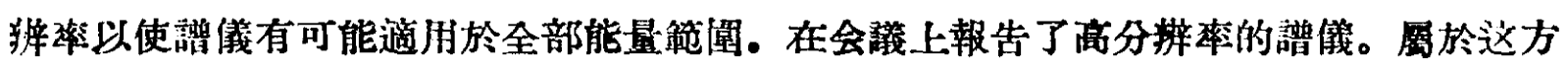
面的有布售克海交实驗室的和苏联的譜義（前者分辢率 0.06 微秒/米, 後者 0.07 微秒/米 和0.12微秒/米)。这些掯儀被用於能量在一万电子伏以下的中子。对於能量較小的中子, 也可以使用按飛躍時間选擇中子的中子諧義。但在这能量範園內, 即由10到 1 电子伏,

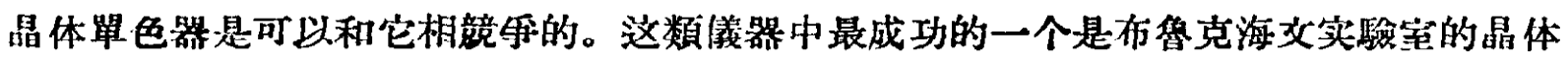
推色器, 其分胁率对 1 电子伏的中子是 $0.4 \%$, 对 50 电子伏的中子是 $2.5 \%$ 。它朋的是铍 的晶体。在中子能量小時, 由於较高次的中子的反射, 应用晶体單色器便不合適了。在这

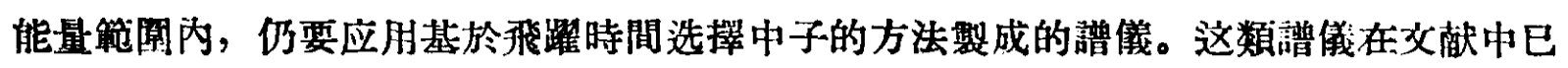
有敍述, 我們將不講了。再回來談一談在幾个电子伏的中子能量範園內工作的譜儀。休 斯在報告中很引人注意地提到, 在美國阿尔阙实驗室正在設計一个分辩時間为 0.035 微 秒/米和 0.02 微秒/米、飛路距离为 60 米的譜儀。艾格尔斯達夫的發言也值得注意, 他報

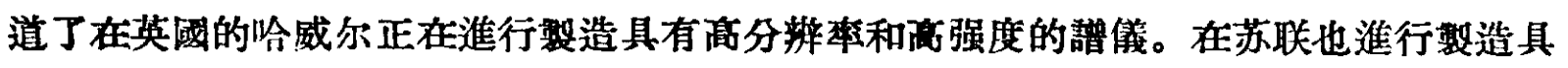

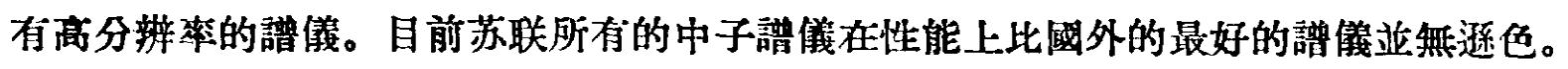

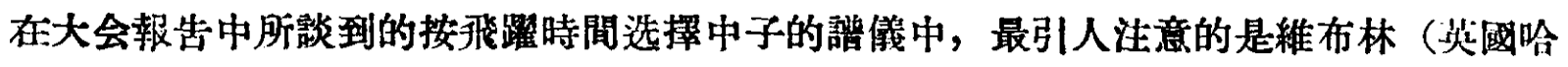
威尔）所報告的工作。为了道生中子脈冲应用了电子能量为 15 百万电子伏、脈冲电流

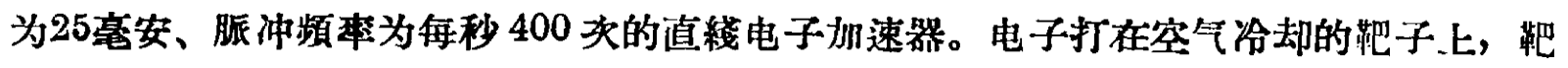
子是用天然鈾做的, 放在鉙慗的凯中, 由靶子放出的中子在石臘中減速. 所得的中子能 
䜊在 1 电子伀以上沂似地服从 $1 / E$ 律。放了 6 組探測器, 距离由 3 到 55 米。在其 他一 系列实驗中，电子打在放在反应堆的相中的特製的距一比。現在利用这个譜儀在能量到

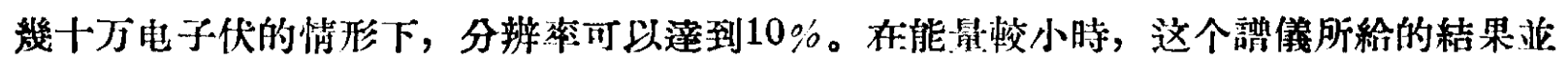
不遜於施用机械截断器於原子堆中子法時所得的結果。这个譜儀具有一系列原則上的优

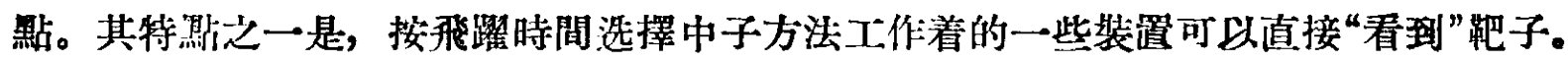
可以使电子束通过長的可動的管子打向靶子，距子放在大的隼䈯中，例如原子堆的柵中。 这种譄儀的最重要的特性是它能够利用对於 $\gamma$ 射線灵䑤的探测器。因为在脈冲之間的射

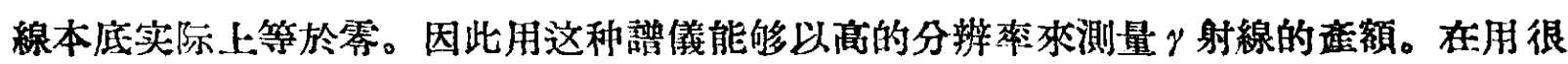
薄的样品 $\left(n \sigma_{0} \ll 1\right)$ 進行測量的条件下，这种实驗能够提供用來分析能級的数据。

驳种諯儀有二个缺點，一个是对中子的最高能量的限制，这是由於最初的 $\gamma$ 射線脈

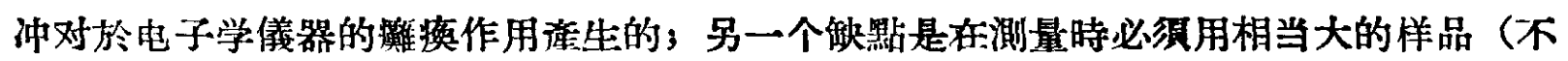
小於 1 点)。

上面所講的譜儀有很远大的前途，它可能使中子的强度篔加好幾个数量級。

在使用按飛躍時間选臎中子的方法的、幾百万地子伏的中子能謭的測量的領域中， 克朗勃格的報告受到了人們的洼意。这个報告講到在十万万分之一秒的時間間隔內的测 量方法。在討論中報告了在苏联淮行的類似的測曈。

沙必罗在教告中提出了中子能譄学方面的十分有独創性的方法, 就是按照中子在大

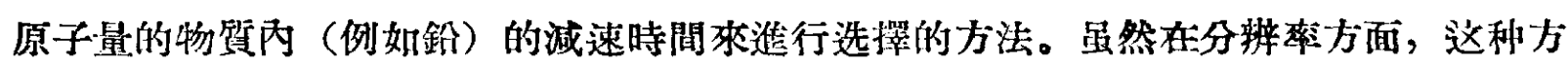
法不能与揬飛躍時間选擇中子的方法相比，但是它有一系列的优點：它有較大的强度， 有可能利用幾十万电子伏的加速器所生的重氢和氛的核反应作为中子源, 以及可以测

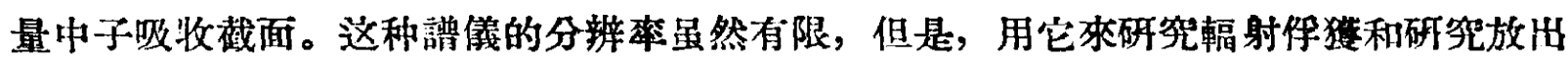
帶电粒子（能量1,000一10,000电子伏）的反应是有利的。

除上面講到的一系列的現在所用的謭儀以外，有一些報告講到利用於脈冲的時間选 擇的侜器。

此外还提到各种能量的中子的探測器。

在用來淮行脈冲的時間选擇和記錄脈冲的儀器中, 現在展泛利用丁近代电子計算机 中所用的存僻器。

中子譄儀的分㸉卒在原則上可以用增加飛躍距离的办法尽量地改善。唯一的也是基 本的限制在於中子源的强度或者对应地來說就是孔道中的計数速率。因此，很明智，不 同能量中子的高效率的探測器的研究在今天受到很大的注意。我們看到的䞶问是利用發 光的晶体、液体和塑料，以及制作和利用記錄俘獾中子後放出的 $y$ 射線的挆测器.

\section{㧴面的測}

在計算鈾堆時，關於核燃料原子核同各种能量中子相互作用的截面的知識是很基本 的东西。在日內瓦会議前这些材料都是最嚴格地保守秘密的。在会議上苏联、英國、美 國和法國提仙了許多有關测量鈾族元素原子㮠截面的報告。我們現在不必去談这些实驗 装置的細管。这些实驗大部分都是用中子譜儀和晶体謭儀作的，中子謭儀根据飛躍時間 选搳中子的原則進行工作。現在各國学者測量的結果都已列入休斯編輯:的中子截面表中。 
把这些測量結果对比一下, 發現这些曲綫彼此符合得很好, 好到在許多情况下, 要想分 辨出來截面曲綫經过的某些实驗默到底是哪个实驗室的都不可能。有趣的是只有把各國

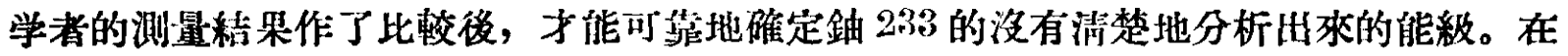
某些情况下苏联和美國学者的工作結果相一致的程度，比美威和垁國的結果間的一较程 度还要嗃些。

因此現在在分裂元素的中子截面方面有了許多彼此一致的数据。鈾 235 的中子截面 湘量得特別准確。確定了所有分裂元素的許多能級的参数（即 $\sigma_{0} 、 、 \Gamma_{n} 、 \Gamma_{f}, \Gamma_{r}$ 和 $\Gamma_{t}$ )。謝 勤对鈾235的測量絬果進行了細心的研究，研究的結果使他能够確定在鈾235各相鄰能級 之間存在千涉現象。这一事实对於原子核分致理論的發展有着重大的意义。

利用中子能級宽度方面的許多資料，我們有可能找到这些筧度的分㛂的經驗規律。

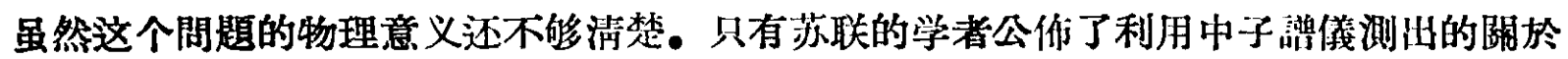

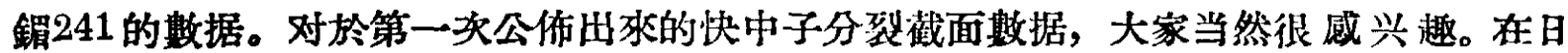
內瓦会議上報道的不分裂元素中子截面数据中，也許最重要的就是对公 151 的測量結果

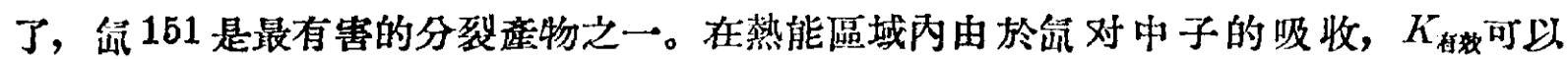
减少 $4 \%$ 左右, 这一點已足以境明問題。在会議上也注意地討論了關於各种截面值的紹 对測量, 这些絕对測量是原子核測量中的根据。哈尔末（美國）作了關於这个問題的報 告。

在日內瓦会議以前, 各國学者探用的是不同的标淮截面敖值, 在可分裂同位素的反应 截面絕对數值方面彼此之間地有些不同。在会議过程中各國代表進行了討諭, 挑选出了 这些截面的一致的数值。这些数值現在已在許多國家的文献中公饰。現在所收集的有關 䅟定同位素共振能區中子截面的大量实驗数据，对於核反应堆的設計有着实际的意义。 这些数据可以直接用來倝算共振效应和温度係数等，同時也刺激了在預言末測过的截面 方面的理論的發展。这种理論的作用和它們在預言截面方面的应用在魏斯考坡夫的報告 中篔作了詳湅的研討.

\section{中子倍增係数}

中子倍堆保数（或可分裂核吸收一个中子發生分裂後，放出的次級中子数） $\eta$ ，在 原子反应堆計算中具有头等重要作用。各國学者在会上都提出了關於测定这个量的報告。 中子的 $\eta$ 可以用各种方法桃測定。其中大部分是根据把具有已知谁面的中子吸收剂和分 裂物質引入反应堆時的反应率变化的比较。在斯比瓦克作的報告中，这种比較在特辋的 指示系中進行。假設由中子能諸分析数据知道了分裂和吸收截面，又知道了分裂時放出 的次粐中子数, 則 $\eta$ 就可以計算出爽。当然, 这样定的 $\eta$ 准確度不福。各國学者測得的 所有熱中子分裂核的 $\eta$ 值彼此很相符。

關於研究中子能量变化時 $\eta$ 的变化的那些報告, 引起人們最大的兴趣。这种兴趣是 由於这些研究很是重要。如果从 $\eta$ 的变化对於反应堆的温度係数的影响着眼。那末中子在 低能範園內時 $\eta$ 的变化確是很重要的, 这个影响与利用反应堆作为能源的問面㨁接有關。 共振能量中子及高能中子的 $\eta$ 值对於建造再生反应堆（从这种反应堆所得到的分裂物質 比維持鏈式反应所消耗的还多）的問題是非常重要的。各國的学者对於建造利用中間能 
中子的再生反应堆都寄以很大的希望，这是由他們的報告裹可以看出來的。可以用各种方 法來研究中子能量变化時 $\eta$ 的变化，在会議中提出的報告內叙述了这些方法中的一部分。 在斯比瓦克的報告和巴列夫斯基的報告中描迡了直接確定 $\eta$ 变化的方法，这方法給 出 $\eta$ 的相对变化的最准確的数值。現在 $\eta$ 的相对变化的测量已達数量級为百万电子伏的中子 能量。關於这样高能的中子的 $\eta$ 值只有苏联代表团作了報道。对於鈈 239 的輻射保獾幾 率及分列幾率的比值 $\alpha$ 所作的測量也引起了㙷然的兴趣。

对於中子能量变化時 $\eta$ 的变化的研究結果指出，对於鈾 235 及鈈 239 而言，在共振隄 的 $\eta$ 值比在熱能區的小，在中子能量洋幾万电子伏時又達到原來的值（即熱能中子的 $\eta$

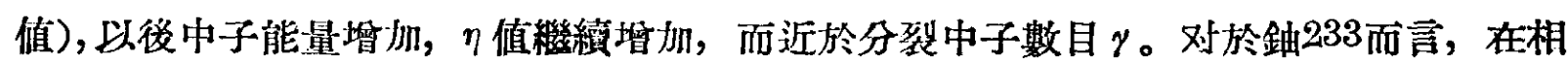
当廣的中子能量䇢園內 $\eta$ 都是不变的, 因此朁可以建成熱中子再生堆, 也可以希望建成 共振中子再生堆。而另一方面，如果要用鈾 235 或踎 239 ，那末大概只有可能造成快中 子再生堆。

\section{紱墢中子}

当分裂後所得到的倅片的激發程度足够發射中子時，其中有一部分中子並不是在分

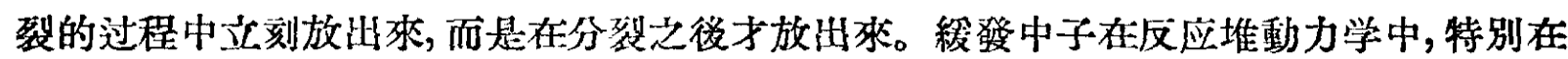
控制鏈式反应的關題中起着重要的作用。在会議上有幾篇關於鈾 238、鈈 239、鈾 233、针

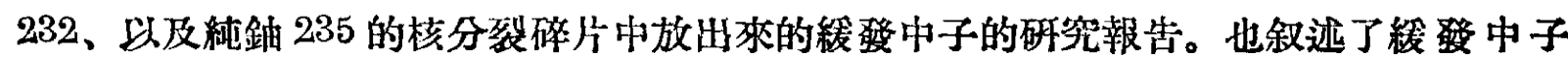
的短寿命的測量技術。

\section{共振吸收皘分}

反应堆建築材料的原子核的中子吸收截面方面的知識，对於㥜算和建立反应堆而 言，其重要性不亞於在分㱟截面方面的知墥。除了用中子讙議測定微分截面外，也常常

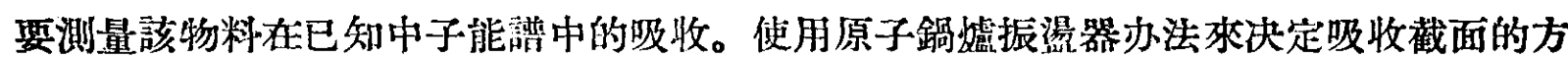
法目前得到了席泛的推度。用这种方法可以决定熱中子的吸收截面和有效共振吸收皘分。 共振吸收皘分是对按照 $1 / \mathrm{E}$ 而变化的中子能譄决定的, $\mathrm{E}$ 是中子的能量, 所謂共振吸收 皘分就是吸收截面（与能量有關）和中子能謭分佈的乘積对能量的積分。除了使用原子 鍋谒振盪器的方法以外, 还可以根据共振中子所引起的激活來测量共振吸收皘分。

在大会上提出了幾篇報告，骼到了所測得的共振吸收皘分，同時也評述了根据原子 核的已知的共振特性來計算共振皘分的可能方法。大家特別注意鈾 238 共振吸收皘分的 計算和测量。这方面可以看到苏联科学家所提出的理論工作。有一篇報告敦可以看到对 这个問題的廣泛的評述。在会議上也提出了關於測量鈾 238 的共振吸收積分的实际工 作。苏联科学院 7 月会議上所報告的工作完全超过了这些工作所得到的結果。在共振吸 收積分的温度效应方面情况也是一样。

有两䈐银告包含着關於不分裂物質共振吸收皘分的知識。其中一箱是苏联的科学家 提比的。在这篇報告中談到用独創性的方法來测量共振䅔分。

現在大家很注意分裂道物对中子的吸收的测量，以及在侙驗性反应堆中具有不同於 熱中子的能湴的中子的吸收的測量。这种測量对於侦用快中子的再生反应堆的計算是特 
別重要的。

\section{物質的娍速特性}

非揖性相互作用截面、要移長度等等數值虽然不像吸收截面那样同系統中的中子寿 命有直接關係，然而它們对於反应堆大小的决定和对於中子能䪪的作用是很叨顯的。建 立再生反应堆時, 關於这些数值的知識是很必需的。侧如, 如果非彈性相互作用的截面 很大，那末中子很快就要失去自己的能量，同時也将減少再生係数; 当中子能是減少㺫 幾十万电子伏以下時， $\alpha$ 增加得相当快。目前非㗊性截面的測量技術弡展得很順利，这 种技術在塔什克的報告中售加以描述。

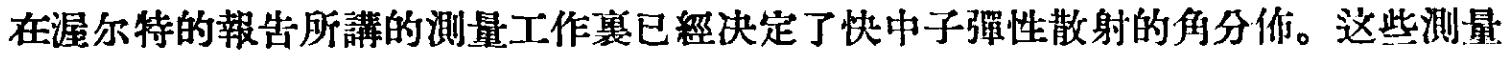
是在中子能量不相同以及原子核的原子量不相同的各种情况下進行的。已得到的丰富財 料允許我們根据已知的角分佈外推而得到合未湘量的原子核的散射角分倠。快中子对鈾 235、鈾 238、钚 239 的彈性散射的角分佈的数据是很有價值的。由於 $\alpha$ 闹能量有相当密 可的關係，非彈性相互作用的截面值更是非常重要。在渥尔特的報告和帕謝契尼克的教 告中也談到了非㽞性相互作用的截面的測量結果。藉助於記錄由於中子非彈性散射耐弡 生的 $\gamma$ 射線來研究快中子和原子核的非彈性相互作用所得到的粘果，比是很有兴趣的。 在这些工作中，不僅測量了 $\gamma$ 射線能譜，而且也測量了激發函数和非彈性相互作用的截 面。

从原子核譜学的覌點來看，後网項工作都是很有兴趣的。因为这些工作可以給出决 於所研究的原子核的低激發能級的知韵。在会莪上提出了一系列關於在水、石黑和铍(通

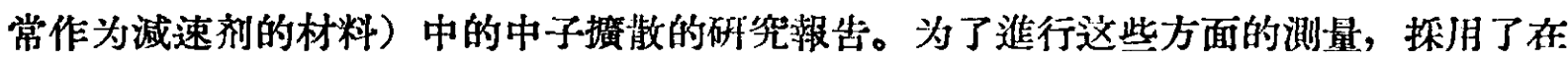
文献中大家所熟知的研究中子穕定分佈的方法，也探用了脉冲中子束的方法。後一測量 方法可直接給出自由中子在各种物筫中的寿命和擴散係数的知識。用这种測量方法測量 控散係数可以隻確到百分之幾。也就是說，它不但比老方法簡單，而且更玔准確。此 外，这种方法还可用以研究中子減速过程的最啳的幾个階段，以及售散係数同中子'所

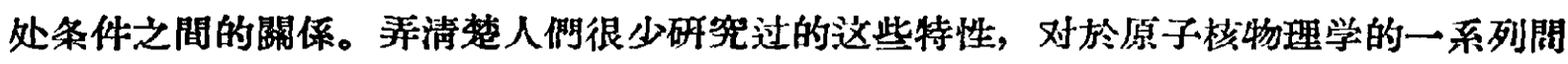
題有很重要的意义。

除了關於熱中子揣散的实驗以外，还報告了在各种物蜇中中子的減速長度或者中子 的寿命的測量結果。

\section{原子核分裂物理学}

如果一种过程提供出使用原子能的可能性, 那末这种过程的物理学無疑地会是很有

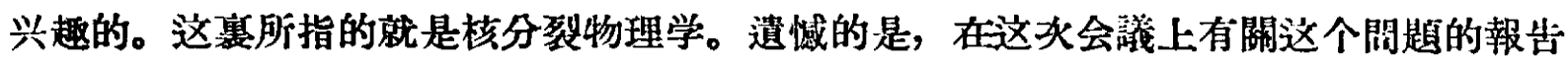
提出得不多。但李奇曼所做的關於核分裂的各种特性的实驗研究工作的論述是值得注意 的。在这論废中引入了用量熱計法測得的核分裂時所放出能量的最新的数据。关於每一 次分裂所座生的次級中子数、这数值的起伏、它闹中子能量的關係等開題的研究，也很

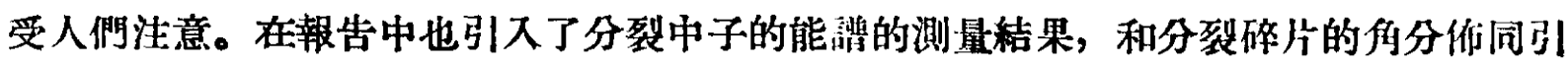
起分裂的中子能量之間的關保的測量結果。 
在会䅈上所提供的有關核分裂的理論工作主要是關於核分裂机構的閏題。应当指出， 到目前为止在这方面虽然有了許多理諭，但是問題还沒有完全弄清楚。

\section{其他工作}

在設計榜反应堆時發生了如何防禦核俘獾中子後所﨎生的或者分裂碎片所放射的中 子和 $\gamma$ 射線的闒題。在許多情况下，例如用重水或者用鈹做为減速剂的反应堆中，从減 速剂光致中子效应这个覌點來看, 分裂碎片放出的 $\gamma$ 射線的產額是很重要的。在挪威已 淮行了分裂碎片所產生的短寿命 $\gamma$ 射線的研究，研究結果見隆得比的報告。

研究核俘獲熱中子後所迹生的 $\gamma$ 射線不僅在防護的覌點上是令人威到兴趣的，同時 它也具有独立的科学上的價值。在会議上報告了在苏联所完成的工作，報告中引入了核 吸收中子時所放出的能量处於自 0.3 百万电子伏到最大能量範圍内的 $\gamma$ 射線的测量結 果。在这样廣的能量範袁內進行 $\gamma$ 射線的測量这还是第一次。

由於有可能从核反应堆獲得大落度的中子束，这就使我們有可能進行中子的 $\beta$ 蛻变 的实驗工作。在苏联完成的其中一个工作已在会議上提出。在这工作中不僅完成了中子 平痤期的測量，也研究了电子和質子的角關联。这个䂰究的結果对於 $\beta$ 蛻变理諭具有原 則性的意义。

地有幾个有關於中子与液体、中子与固体的相互作用的研究以及有关於慢中子的非 匴性相互作用的研究的報出。

有很多報告談到方法上的問題，这对於在实驗原子㮠物理学的各个領域中工作的軎 家們是非常有兴趣的。

在結束我的䜷道前，还希望再强調一次國际会議在原子核物理学未來發展.上的巨大 意义。在会上听取了許多有趣的報告和報道。許多重要的数据已成了全体物理学家的財 詹。会上各國的学者之間建立了个別接触。会議已成为在許多國家內促進原子核物理学 的發展的巨大因素。

\section{[上接 80 頁]}

2. 逐漸將國內現有的少数人类学家適当地集中於科学院。

3. 在北京大学、复旦大学等生物系內增添人类学镋業，大力培养幹部。

4. 增添必要的設备, 如圖書、仪器、标本、模型等。

5. 在第二个五年計划內，首先在北京，然后再在各大城市逐漸建立人类学博物舘。 6. 加强科学院与衛生部門、体育部門、國防部門、工業部門和民族学院等的联系。

总之，人类学的研究有着巨大的科学意义和实用价值。人类学的知識是建立唯物主 义世界覌所必不可缺的一部分。人类学, 正如我們偉大的革命導师恩格斯在 “自然辩証 法”（150 页）中所指出的，“还要繼續詳細地研究和閔明”，而中國在这方面是具有特殊有 利的条件的。因此，在新中國的科学研究机構中，人类学应該佔有它应有的地位! 